Supplement of Biogeosciences, 17, 3165-3182, 2020

https://doi.org/10.5194/bg-17-3165-2020-supplement

(C) Author(s) 2020. This work is distributed under

the Creative Commons Attribution 4.0 License.

(c) (1)

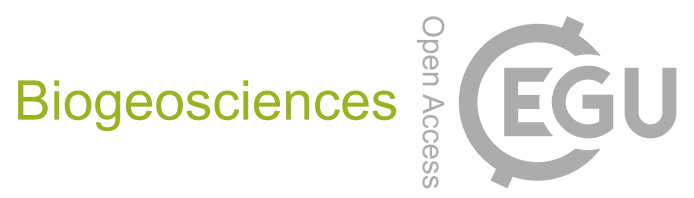

Supplement of

\title{
Bottom-water deoxygenation at the Peruvian margin during the last deglacia- tion recorded by benthic foraminifera
}

\section{Zeynep Erdem et al.}

Correspondence to: Zeynep Erdem (zeynep.erdem@nioz.nl)

The copyright of individual parts of the supplement might differ from the CC BY 4.0 License. 


\section{Supplementary Information: Figures and Tables}

Figure S.1: Graphs showing the relative percentage (\% total calcareous) distributions of the abundant living benthic foraminifera.

Figure S.2: $Q$-mode hierarchical cluster analysis (UPGMA Bray-Curtis similarity index) applied on living taxa.

Figure S.3: Canonical Correspondence Analysis (CCA) results applied to living foraminifera census dataset. Blue dots indicate the species and crosses indicate the samples. Abundant species included in the quantification approach together with species mentioned in the text are shown in bold and with their names.

Figure S.4: Canonical Correspondence Analysis (CCA) results applied to reduced dataset

Table S.1: Results of bottom-water oxygenation estimations $\left(\left[\mathrm{O}_{2}\right]_{\mathrm{BW}}\right)$ in 3 sediment cores with the core depth and calibrated age point information.

Supplementary Information: Information on polynomial transfer function and description to calculations

Supplementary Information: Links to open access datasets related to this study 
Figure S.1. Graph showing the relative percentage (\% total calcareous) distributions of abundant living benthic foraminifera. X-axis indicates the stations (water depth $(\mathrm{m})$ ) which are grouped according to the prevailing bottom-water-oxygen concentrations (black for microxic $(<5 \mu \mathrm{mol} / \mathrm{kg})$, orange for dysoxic $(5-45 \mu \mathrm{mol} / \mathrm{kg})$, green for oxic $(>45 \mu \mathrm{mol} / \mathrm{kg}))$.

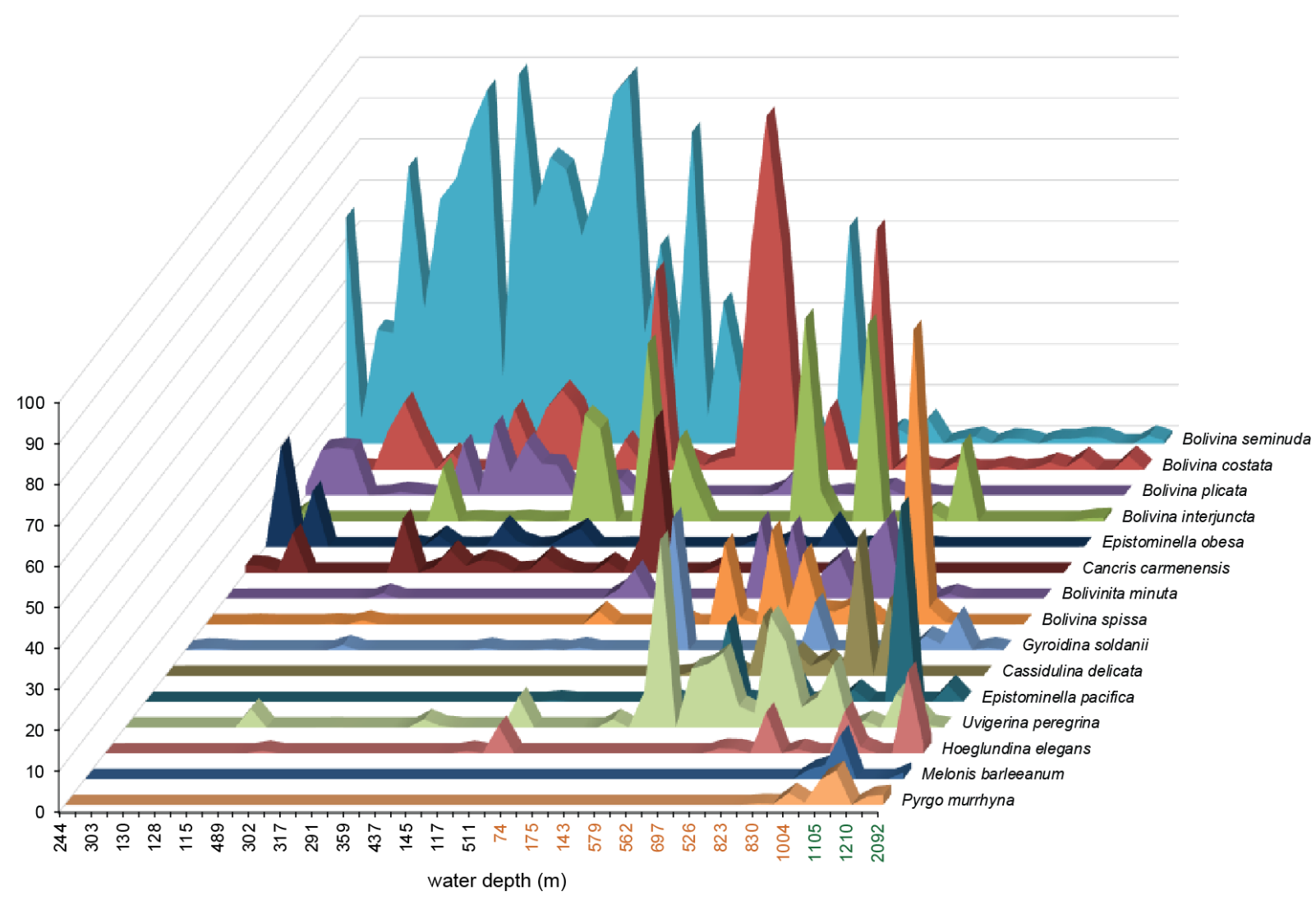


Figure S.2: $Q$-mode hierarchical cluster analysis (UPGMA Bray-Curtis similarity index) applied on living taxa.

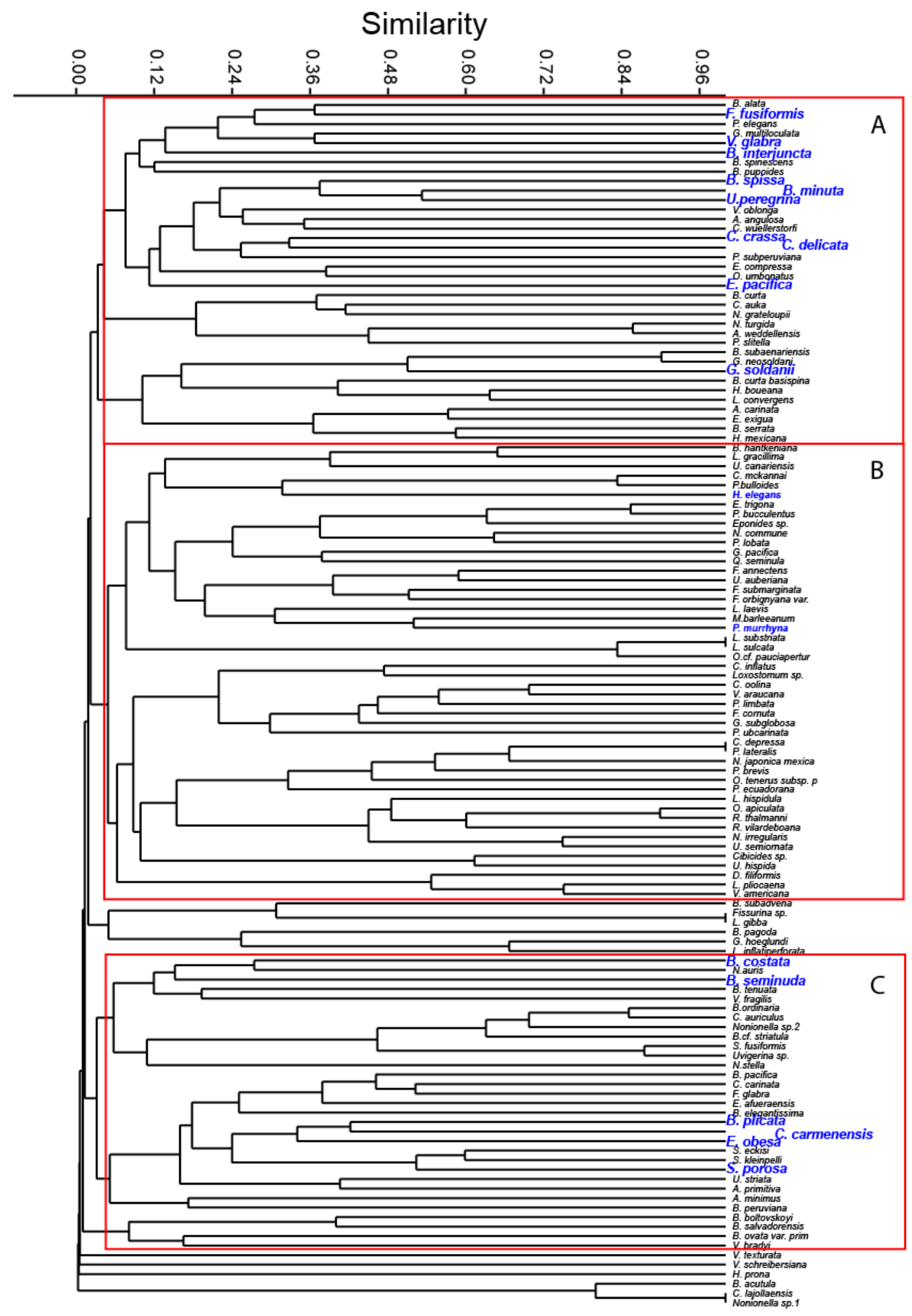


Figure S.3: Canonical Correspondence Analysis (CCA) results applied to living foraminifera census dataset. Blue dots indicate the species and crosses indicate the samples. Abundant species included in the quantification approach together with species mentioned in the text are shown in bold and with their names.

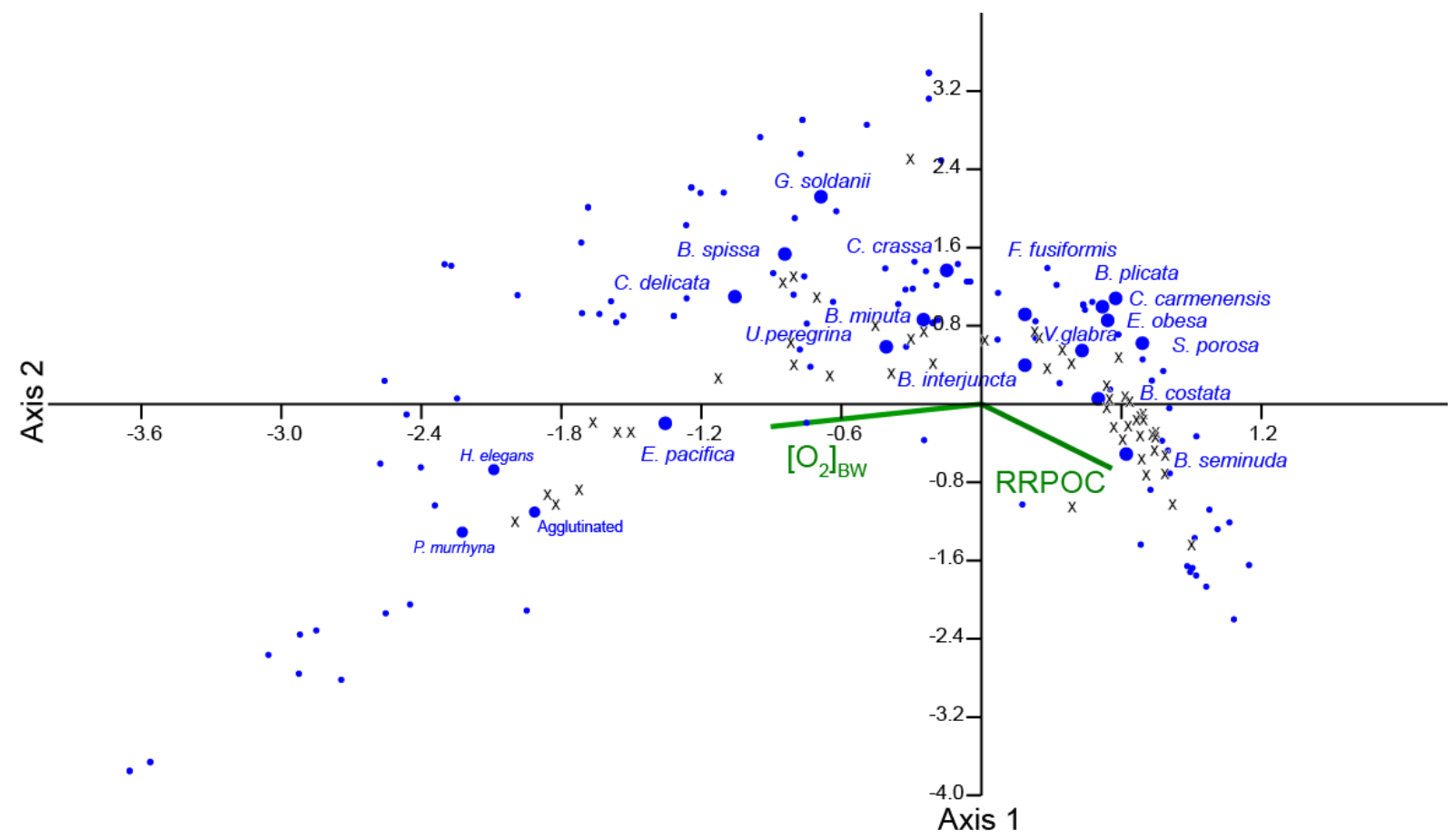

Figure S.4: Canonical Correspondence Analysis (CCA) results applied to reduced dataset (the most abundant 16 species) considered in this study. Blue dots indicate the species and black dots indicate the sample locations. Both in census dataset and reduced dataset certain species showed similar relationship with $\left[\mathrm{O}_{2}\right]_{\mathrm{BW}}$. For example: E. pacifica showing a positive relation with increasing oxygen whereas $B$. plicata group in with similar species showing a negative relation with oxygen concentrations. CCA results also show that the 'oxic' samples are not well represented in this dataset (fewer sampling stations fall positive side of the $\left[\mathrm{O}_{2}\right]_{\mathrm{BW}}$.

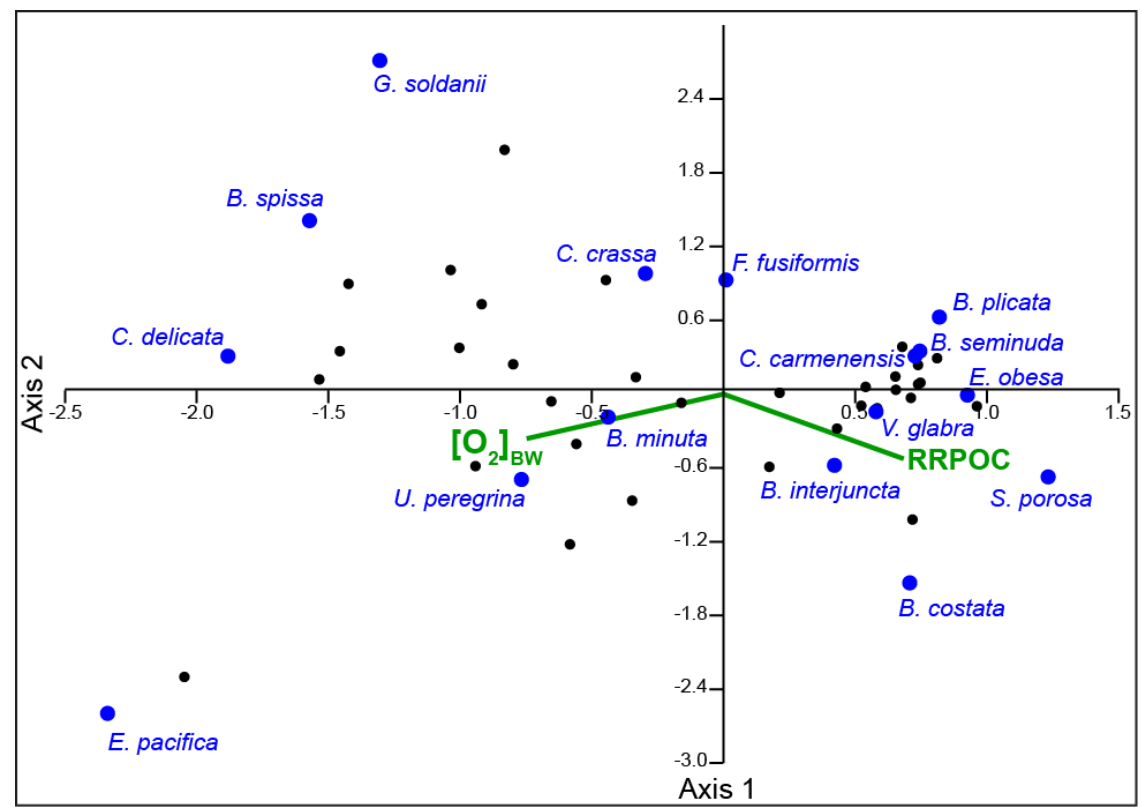


Supplementary information Table 1. Results of bottom-water oxygenation estimations $\left(\left[\mathrm{O}_{2}\right]_{\mathrm{BW}}\right)$ in 3 sediment cores with the core depth and calibrated age point information.

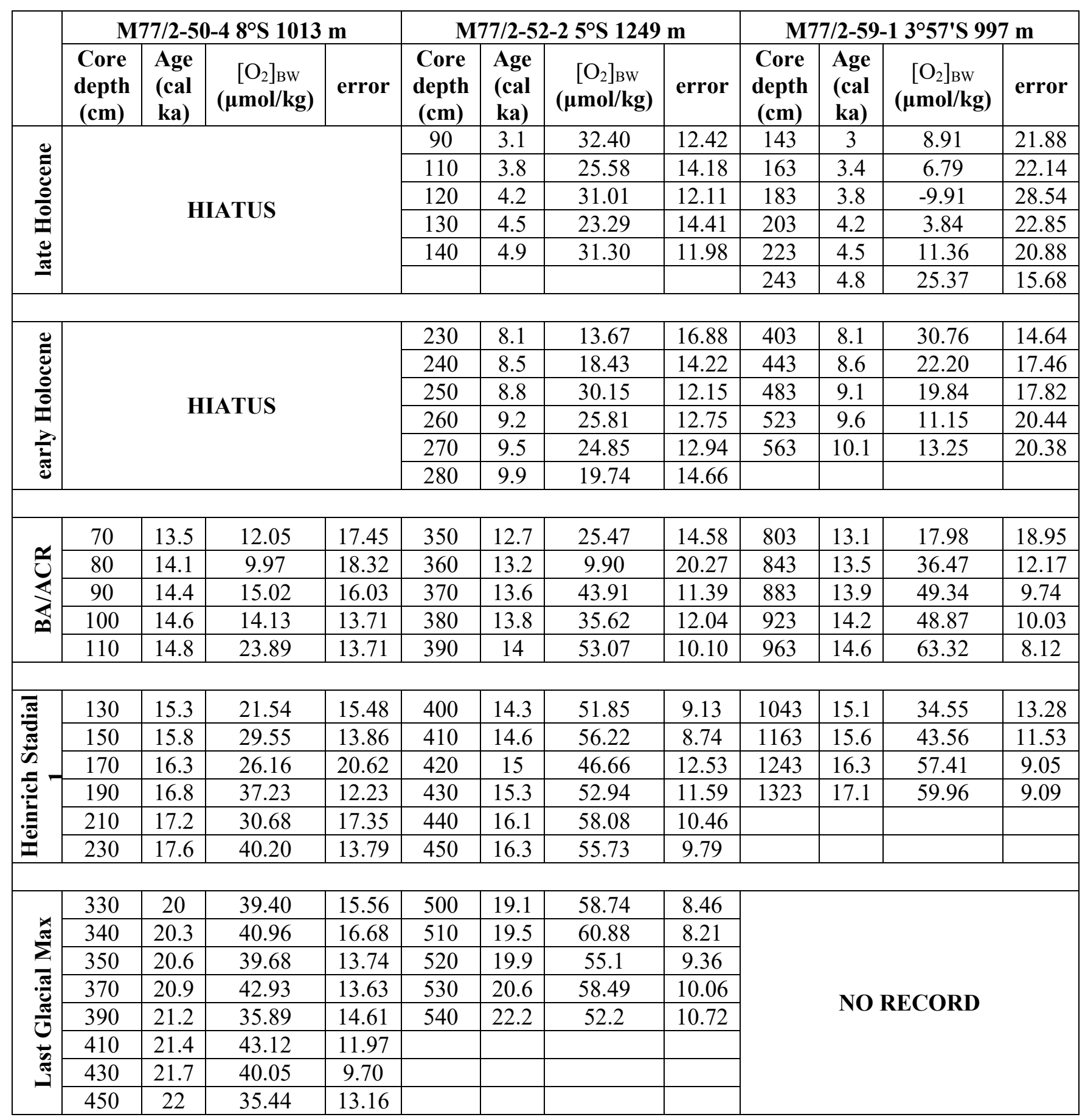




\section{Information on polynomial transfer function and description to calculations}

The polynomial transfer function is given in the form of eq.1:

$$
\text { Eq.1: } \quad x=C+\sum R \mathrm{Co}_{n} \cdot \%_{n}
$$

where $\mathrm{x}$ is the environmental variable that should be reconstructed with the transfer function (in this case $\left[\mathrm{O}_{2}\right]_{\mathrm{BW}}$ or RRPOC); $\mathrm{C}$ is a constant from the multiple regression (tab. 5); $\mathrm{RCo}_{\mathrm{n}}$ is the regression coefficient for the foraminiferal species $n(t a b .5)$; and $\%_{n}$ is the percentage of the foraminiferal species $n$ within the assemblage.

For the calculation of the errors for $\mathrm{x}$ (i.e. $\left[\mathrm{O}_{2}\right]_{\mathrm{BW}}$ or RRPOC) a complete error propagation has been done including the $1 \sigma$ errors of all species within the polynomial transfer function. The error propagation has been applied to the polynomial transfer function (Eq. 1) in the form of equation Eq. 2:

$$
\text { Eq.2: } \quad \sigma_{x}=\sqrt{\left(\frac{\partial x}{\partial C} \cdot \sigma_{C}\right)^{2}+\sum\left(\frac{\partial x}{\partial R C \sigma_{n}} \cdot \sigma_{R C \sigma_{n}}\right)^{2}+\sum\left(\frac{\partial x}{\partial q_{6_{n}}} \cdot \sigma_{9 b_{n}}\right)^{2}}
$$

where $\sigma_{\mathrm{x}}$ is the standard deviation $(1 \mathrm{sd})$ of the environmental variable $\mathrm{x}$ (i.e. $\left[\mathrm{O}_{2}\right]_{\mathrm{BW}}$ or RRPOC) $\sigma_{\mathrm{C}}$ is the error of the constant $\mathrm{C}(\mathrm{tab} .5) ; \sigma_{R C \sigma_{\mathrm{n}}}$ is the standard error for the regression coefficient RCo for the foraminiferal species $\mathrm{n}(\mathrm{tab} .5)$; and $\sigma_{96_{\mathrm{n}}}$ is the standard error for $\%_{\mathrm{n}}$. Solution of the derivatives in Eq.2 results in Eq.3:

$$
\text { Eq.3: } \quad \sigma_{x}=\sqrt{\sigma_{C}{ }^{2}+\Sigma\left(\%_{n} \cdot \sigma_{R C o_{n}}\right)^{2}+\Sigma\left(R C o_{n} \cdot \sigma_{96_{n}}\right)^{2}}
$$

We have to state that we neglect the last term in equations 2 and 3 because we do not know $\sigma_{90_{\mathrm{n}}}$, since three replicates would be necessary for each sample to determine this error. In this study all downcore samples were counted by a single investigator. Previous studies showed that population densities of sample replicates, which have been picked dry by a single investigator had an accuracy $(1 \sigma)$ of $\pm 2 \%$ (Schönfeld et al., 2013). Thus, in comparison with the high $\sigma_{\mathrm{C}}$ and $\sigma_{R C o_{\mathrm{n}}}(\mathrm{tab} .5)$ the error $\%_{\mathrm{n}}$ should be negligible. Nevertheless, the proportions of frequent species within the same assemblage may differ by $2-7 \%$ between different picking modes or laboratories (Schönfeld et al., 2013). 


\section{Links to open access datasets related to this study}

Living benthic foraminifera (rose Bengal stained) dataset can be reached through the following link: https://doi.pangaea.de/10.1594/PANGAEA.901840

Radiocarbon ages of core M77/2-52-2: https://doi.pangaea.de/10.1594/PANGAEA.877426

Radiocarbon ages of core M77/2-50-4: https://doi.pangaea.de/10.1594/PANGAEA.902616

\section{Reference}

Schönfeld, J., Golikova, E., Korsun, S., Spezzaferri, S.: The Helgoland Experiment - assessing the influence of methodologies on Recent benthic foraminiferal assemblage composition, Journal of Micropaleontology, 32, 161-182, doi: 10.1144/jmpaleo2012-022, 2013. 